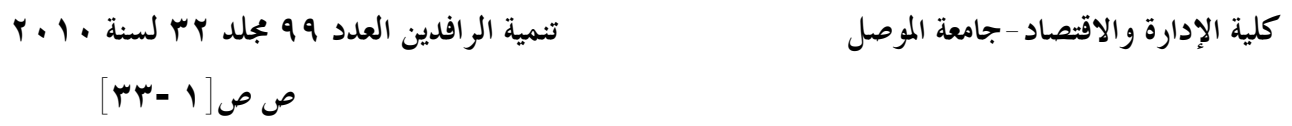

azza_master

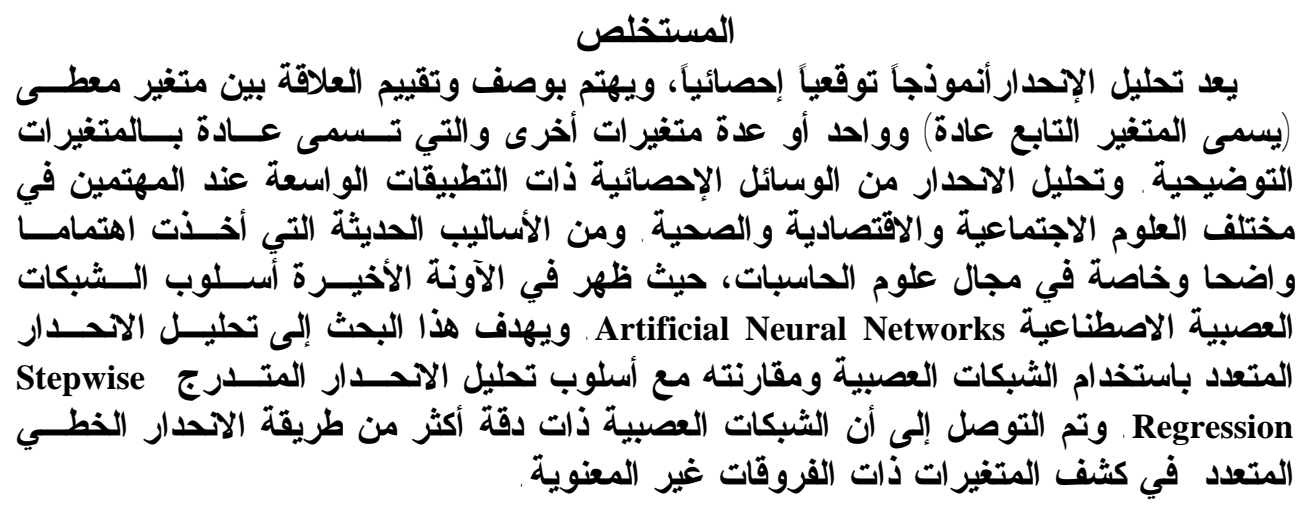

\title{
Using Artificial Neural Networks in Multiple Linear Regression
}

Nadwa Kh. Rashad

Assistant Lecturer

Department of Management Information

Systems

University of Mosul
Azza H. Zaki

Assistant Lecturer

Department of Statistics and Information

University of Mosul

azza_master@yahoo.com

\begin{abstract}
The regression analysis is considered as an expectative and statistical model. It concerns with the description and standardization of the relationship between a given variable which is called (dependent variable) and other variables called independent variables. Regression analysis is one of the statistical methods that have wide applications to those concerning with many different societies, economic and health sciences. One of the modern methods that clearly concerned with the computer field which has appeared these
\end{abstract}


رشاد وزكي[r]

days is Artificial Neural Networks. The aim of this research is the comparison between Neural Networks Method with multiple regressions using stepwise regression method.

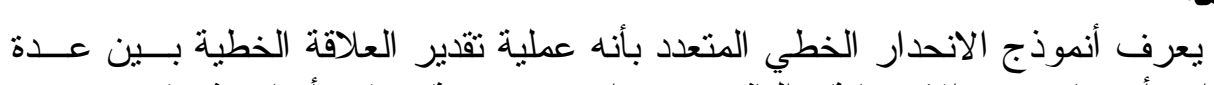
المقدمة

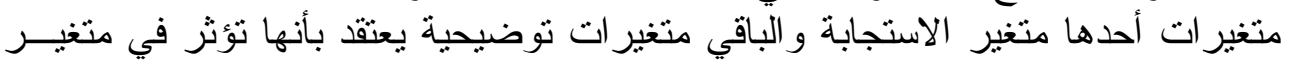

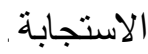

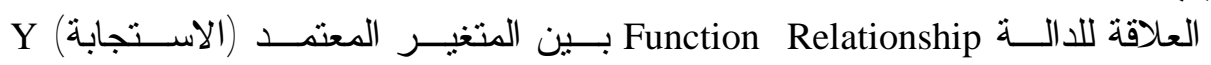
و المتغير ات التوضيحية (X) الاتية (شربجي، (191) ) $Y_{i}=\beta_{o}+\beta_{1} X_{i 1}+\beta_{2} X_{i 2}+\ldots+\beta_{m} X_{i m}+U_{i}$

$Y_{i}=\beta_{0}+\sum_{j=1}^{m} \beta_{j} X_{i j}+U_{i} \quad, i=1,2, \ldots, n ; j=1,2, \ldots, m$

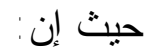

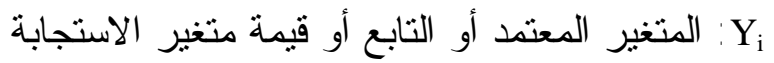

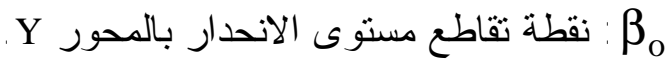
海 $\beta_{1}, \beta_{2}, \ldots, \beta_{m}$ U

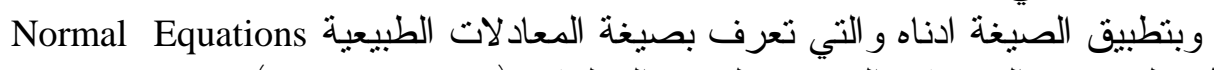
نحصل على مقدر المربعات الصغخرى لمتجه المعلمات (شربجي، (1919 ) . $\hat{\beta}=\left(X^{\prime} X\right)^{-1} X^{\prime} Y$

$$
\begin{aligned}
& \text { حيث ان } \\
& \text { Information Matrix مصفوفة مربعة تدعى مصفوفة المعلومات : X'X } \\
& \text { متجه لحاصل ضرب المتغير ات التوضيحية في متغير الاستجابة : X' ' X } \\
& \mathrm{X}^{\prime} \mathrm{X}=\left[\begin{array}{lcccc}
\mathrm{n} & \sum \mathrm{x}_{\mathrm{i} 1} & \sum \mathrm{x}_{\mathrm{i} 2} & \ldots & \sum \mathrm{x}_{\mathrm{im}} \\
\sum \mathrm{x}_{\mathrm{i} 1} & \sum \mathrm{x}_{\mathrm{i} 1}^{2} & \sum \mathrm{x}_{\mathrm{i} 1} \mathrm{x}_{\mathrm{i} 2} & \cdots & \sum \mathrm{x}_{\mathrm{i} 1} \mathrm{x}_{\mathrm{im}} \\
\cdot & \cdot & \cdot & \cdots & \cdot \\
\sum \mathrm{x}_{\mathrm{im}} & \sum \mathrm{x}_{\mathrm{im}} \mathrm{x}_{\mathrm{i} 1} & \sum \mathrm{x}_{\mathrm{im}} \mathrm{x}_{\mathrm{i} 2} & \cdots & \sum \mathrm{x}_{\mathrm{im}}^{2}
\end{array}\right]
\end{aligned}
$$


$\mathrm{X}^{\prime} \mathrm{Y}=\left[\begin{array}{l}\sum \mathrm{Y}_{\mathrm{i}} \\ \sum \mathrm{Y}_{\mathrm{i}} \mathrm{X}_{\mathrm{i} 1} \\ \sum \mathrm{Y}_{\mathrm{i}} \mathrm{X}_{\mathrm{i} 2} \\ \cdot \\ \\ \sum \mathrm{Y}_{\mathrm{i}} \mathrm{X}_{\mathrm{im}}\end{array}\right]$

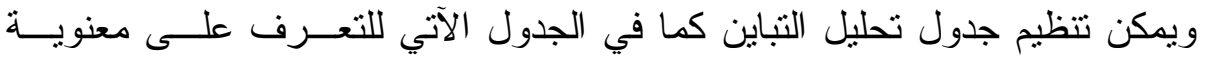

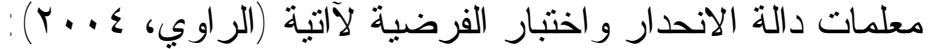

$\mathrm{H}_{\mathrm{o}}: \beta_{1}=\beta_{2}=\ldots=\beta_{\mathrm{m}}$

$\mathrm{H}_{1}: \beta_{1} \neq \beta_{2} \neq \ldots \neq \beta_{\mathrm{m}}$

جدول تحليل التباين للاحدار المتعدد

\begin{tabular}{|c|c|c|c|c|}
\hline S.o.V. & d.f & S.S & MS & F.cal. \\
\hline $\mathrm{R}\left(\mathrm{X}_{1}, \mathrm{X}_{2}, \ldots, \mathrm{X}_{\mathrm{m}}\right)$ & $\mathrm{m}$ & SSR & MSR & $\frac{\mathrm{MSR}}{\mathrm{MSe}}$ \\
\hline Error $\left(\mathrm{X}_{1}, \mathrm{X}_{2}, \ldots, \mathrm{X}_{\mathrm{m}}\right)$ & $\mathrm{n}-\mathrm{m}-1$ & SSe & MSe & \\
\hline Total & $\mathrm{n}-1$ & SST & & \\
\hline
\end{tabular}

حيث إن: (مير

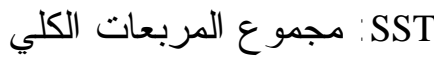

$\mathrm{SST}=\mathrm{S}_{\mathrm{YY}}$

SST $=y^{\prime} y-\frac{\left(\sum y_{i}\right)^{2}}{n}$

$\mathrm{SST}=\sum \mathrm{y}_{\mathrm{i}}^{2}-\frac{\left(\sum \mathrm{y}_{\mathrm{i}}\right)^{2}}{\mathrm{n}}$

$\operatorname{SSR}=\underline{\beta^{\prime} x^{\prime} y}-\frac{\left(\sum y_{i}\right)^{2}}{n}$

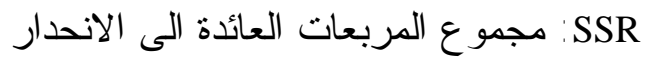


رشاد وزكي[؛]

$$
=\left[\beta_{o} \beta_{1} \ldots \beta_{m}\right]\left[\begin{array}{l}
\sum_{i=1}^{n} y_{i} \\
\sum_{i=1}^{n} x_{i 1} y_{i} \\
\cdot \\
\sum_{i=1}^{n} x_{i m} y_{i}
\end{array}\right]-\frac{\left(\sum y_{i}\right)^{2}}{n}
$$

$\mathrm{MSR}=\frac{\mathrm{SSR}}{\mathrm{m}}$

MSR

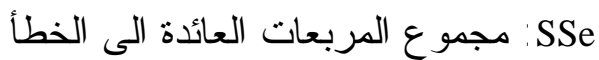

$\mathrm{MSe}=\frac{\mathrm{SSe}}{\mathrm{n}-\mathrm{m}-1}$

فإذا كانت قيمة F المحسوبة مساوية أو أكبر من قيمة F الجدوليــة تحـــت مسستوى

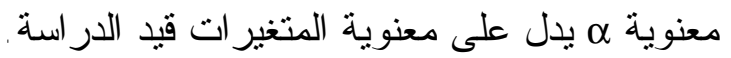

طريقة الاحدار المتدرج Stepwise Regression Procedure

تستخدم هذه الطريقة في تحليل الانحدار المتعدد لانتخاب المتغيرات ذات التئ التأثير

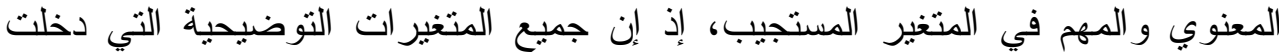

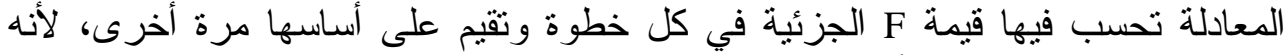

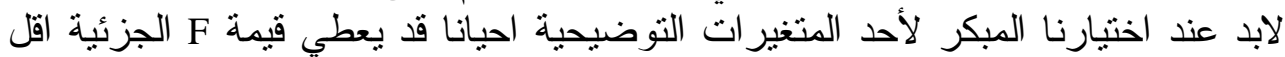

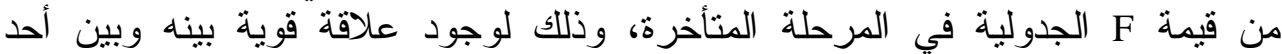

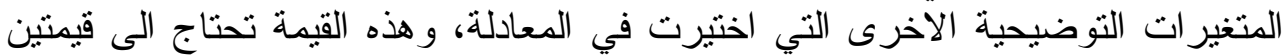

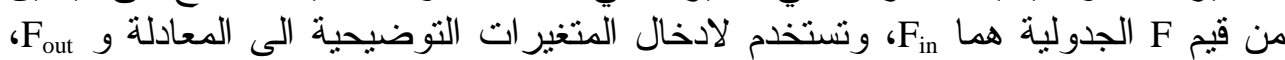

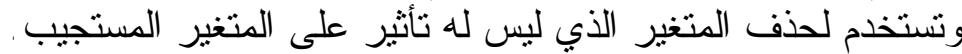

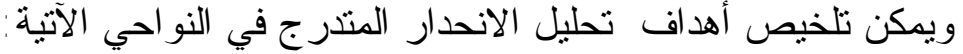

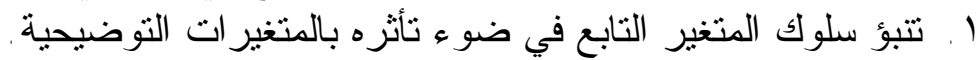

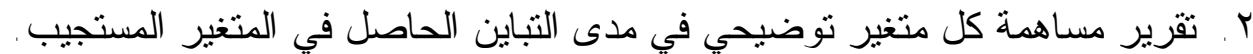

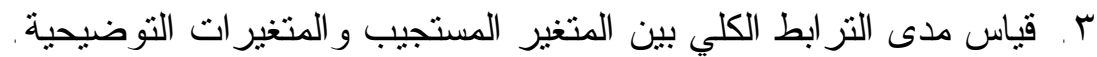
ع. إجراء سلسلة من الاختبار ات الفرضية لأي من العلاقات المشار إليها في النقاط الئاط

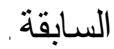

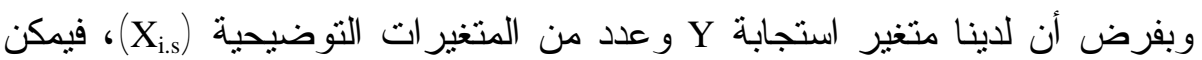

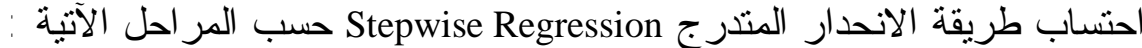

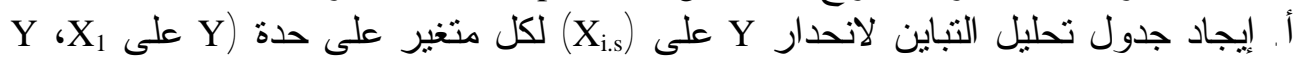

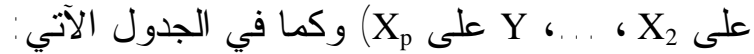




\begin{tabular}{|c|c|c|c|c|}
\hline S.o.V. & d.f & S.S & MS & F.cal. \\
\hline $\mathrm{R}\left(\mathrm{X}_{1}\right)$ & $\mathrm{p}$ & $\mathrm{B}^{\prime} \mathrm{X}^{\prime} \mathrm{Y}-\mathrm{n} \overline{\mathrm{Y}}^{2}$ & $\left(\mathrm{SSR} * \mathrm{X}_{1}\right) / \mathrm{d} . \mathrm{f}$ & $\frac{\mathrm{MSR}}{\mathrm{MSe}}$ \\
\hline Error $\left(\mathrm{X}_{\mathrm{i}}\right)$ & $\mathrm{n}-\mathrm{p}-1$ & $\mathrm{Y}^{\prime} \mathrm{Y}-\mathrm{B}^{\prime} \mathrm{X}^{\prime} \mathrm{Y}$ & $\mathrm{SSe} /$ d.f. & \\
\hline Total & $\mathrm{n}-1$ & $\mathrm{Y}^{\prime} \mathrm{Y}-\mathrm{n}^{2}$ & & \\
\hline
\end{tabular}

$$
\text { حيث : }
$$

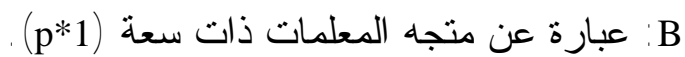

(p*n) من المتغير ات التوضيحية.

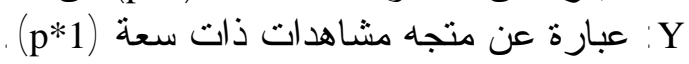

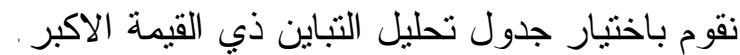

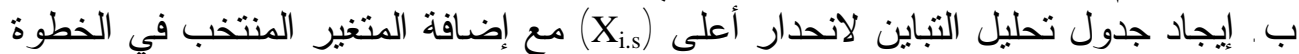
(أ) وبحسب جدول تحليل التباين الآتي:

\begin{tabular}{|c|c|c|c|c|}
\hline S.o.V. & d.f & S.S & MS & F.cal. \\
\hline$R\left(X_{i}, X_{k}\right)$ & $p$ & $B^{\prime} X^{\prime} Y-n \bar{Y}^{2}$ & & \\
\hline$R\left(X_{k}\right)$ & 1 & $B^{\prime} X^{\prime} Y-n \bar{Y}^{2}$ & & \\
\hline$R\left(X_{i} / X_{k}\right)$ & 1 & $B^{\prime} X^{\prime} Y-n^{2}$ & $\frac{S S R\left(X_{i} / X_{k}\right)}{d . f}$ & $\frac{M S R\left(X_{i} / X_{k}\right)}{M S e}$ \\
\hline Error $\left(X_{i}, X_{k}\right)$ & $n-p$ & $Y^{\prime} Y-B^{\prime} X^{\prime} Y$ & $\frac{S S e}{d . f}$ & \\
\hline Total & $n-1$ & $Y^{\prime} Y-n \bar{Y}^{2}$ & & \\
\hline
\end{tabular}

حيث إن:

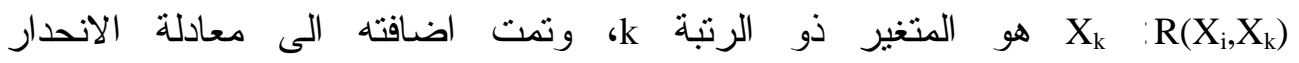

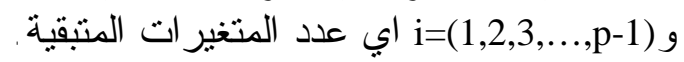

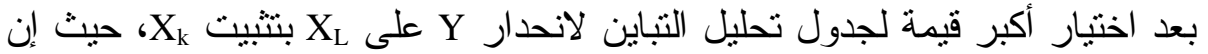

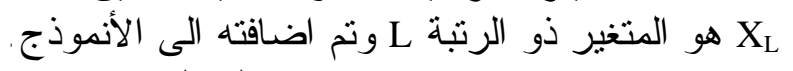

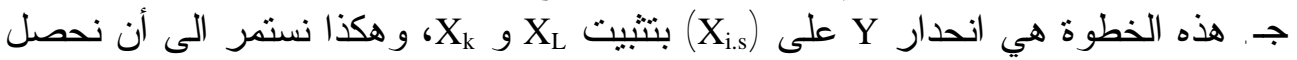

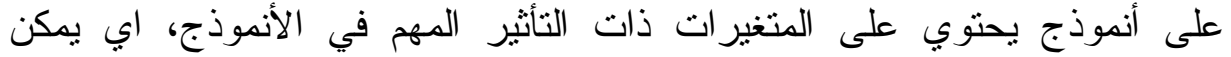
استخدام هذه المتغيرات في الحصول على على تمبيز افضل بين مجتمعين (الراوي، () (19人V

و الثكل الآتي يبين خوارزمية الانحدار المتدرج (Wikipedia,2008) 
رشاد وزكي[י]

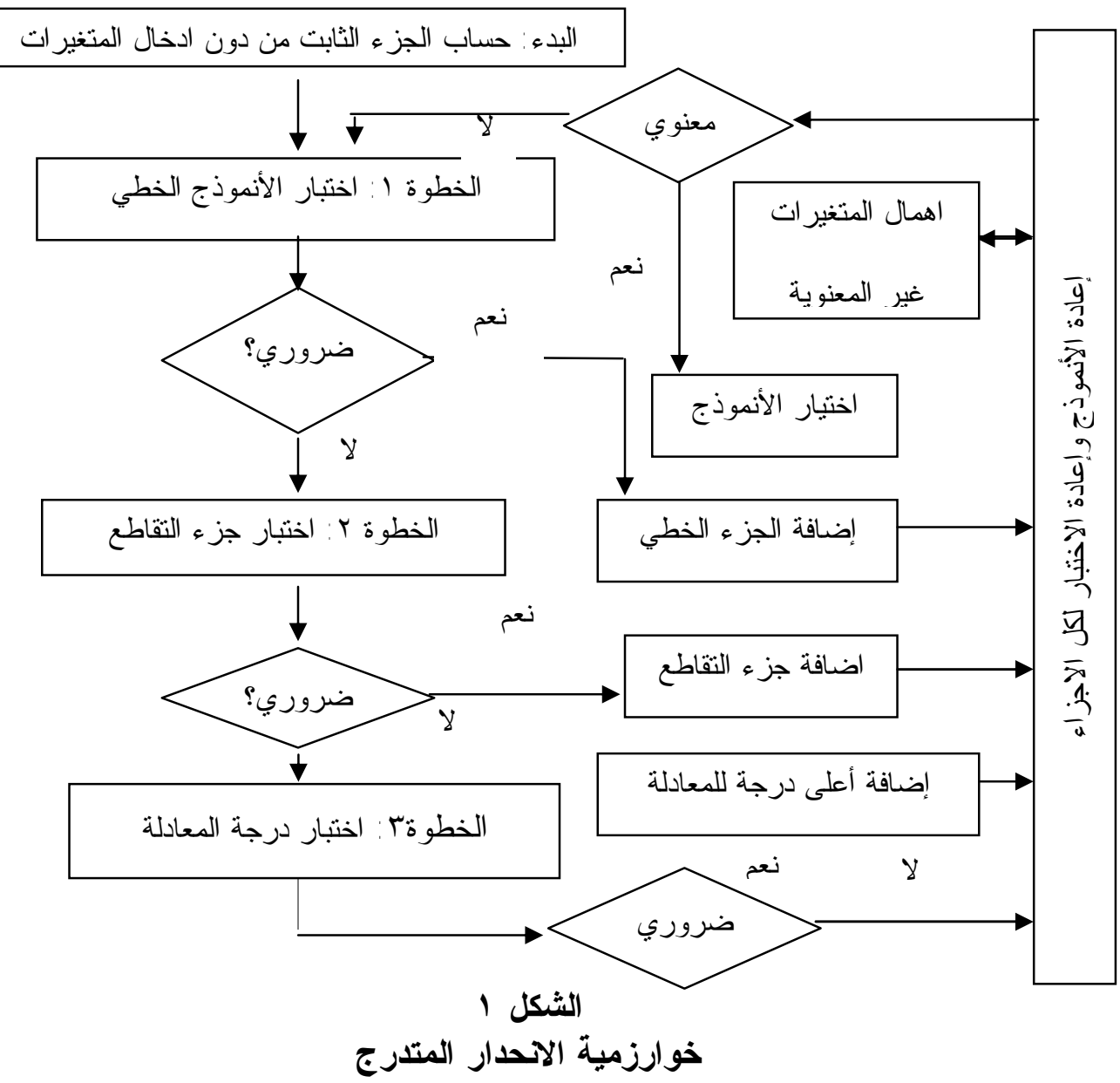

\section{Artificial Neural Networks الثبكات العصبية الاصطناعية}

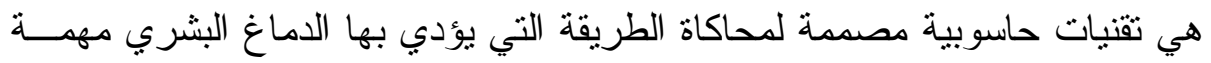

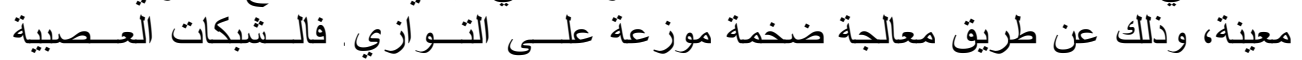

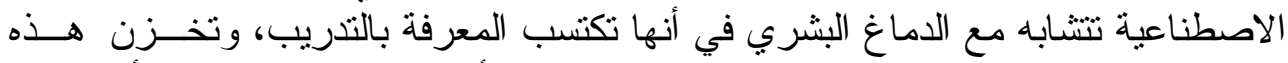

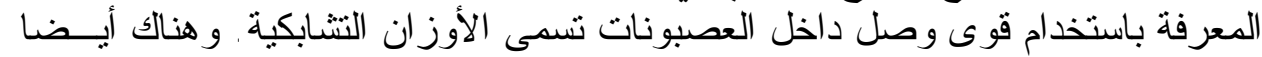

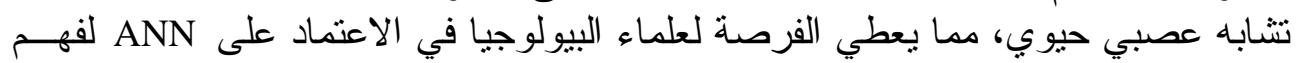
تطور الظو اهر الحيوية.

مكونات الشبكة العصبية الاصطناعية 
تتكون الثبكات العصبية من مجموعة من وحدات المعالجة، ويسمى أحدها عصبون،

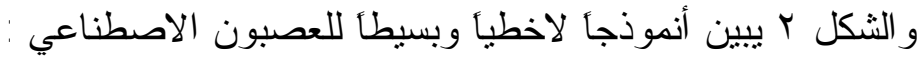

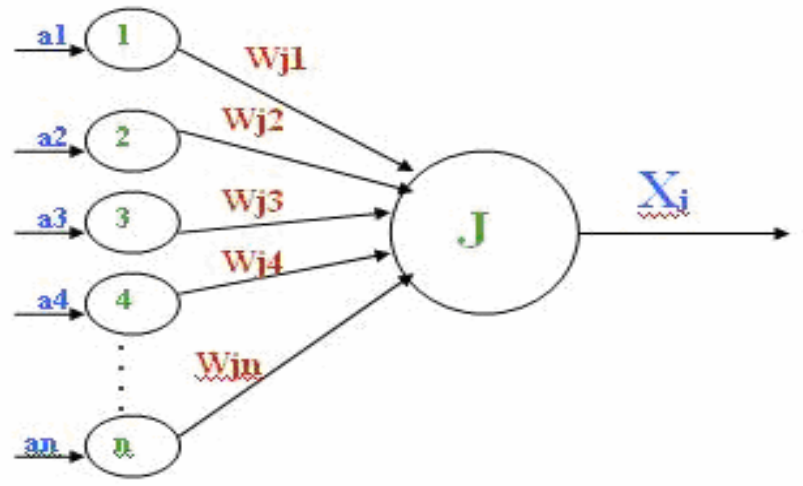

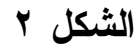

خلية عصبية اصطناعية

كما إن للإنسان وحدات إدخال توصله بالعالم الخارجي و هي حو اسه الخمس، فكذللك الثبكات العصبية تحتاج لوحدات إدخال . ووحدات معالجة يتم فيها عمليات حسابية تضبط

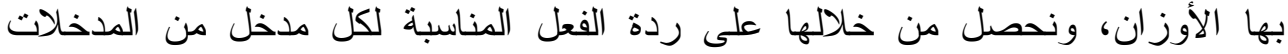

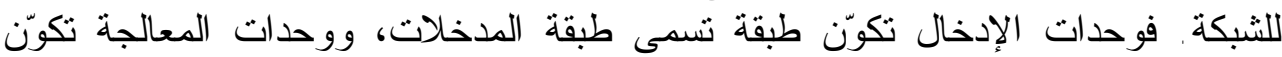
طبقة المعالجة وهي التي تخرج نو اتج الثبكة. وبين كل طبقة من هذه الطبقات هنات هناك طبقة

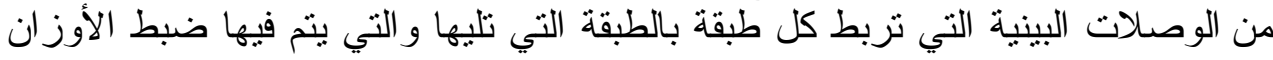

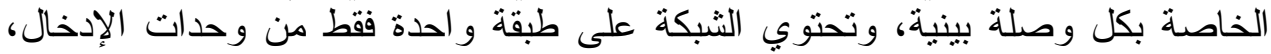

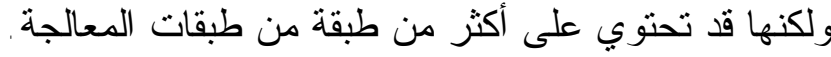

\section{البنية المعمارية للشبكات العصبية}

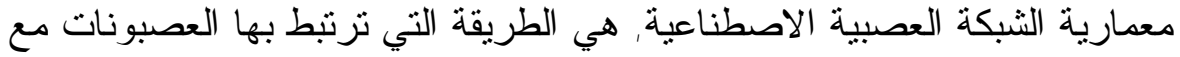

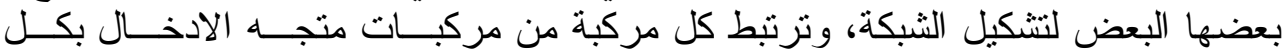

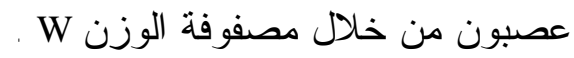
كل عصبون يحوي وحدة مجموع تقوم بجمع الادخال الموزون العدون مع الإز احة لتشكيل

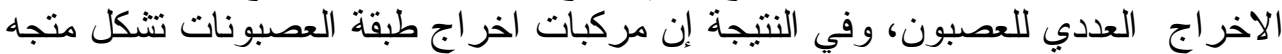
الاخر اج مركبات متجه الادخال تذخل إلى الثبكة من خلال مصفوفة الإنة الأوز ان الآتية: 


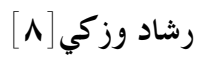

$$
\mathbf{W}=\left[\begin{array}{llll}
w_{1,1} & w_{1,2} & \cdots & w_{1, R} \\
w_{2,1} & w_{2,2} & \cdots & w_{2, R} \\
w_{S, 1} & w_{S, 2} & \cdots & w_{S, R}
\end{array}\right]
$$

الثبكة ذات الطبقات المتعددة الأمامية

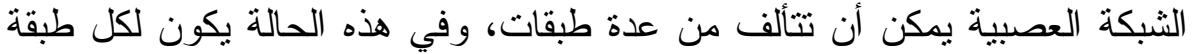

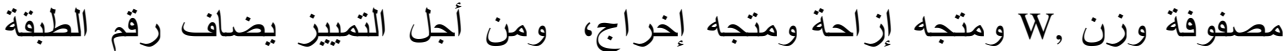

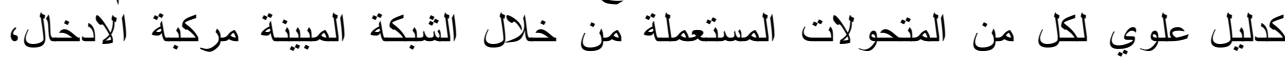

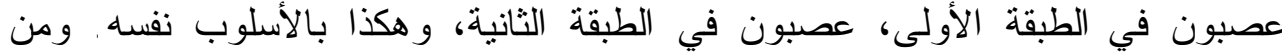

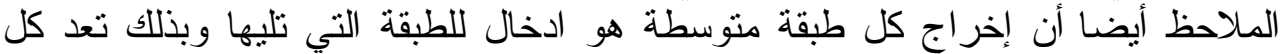

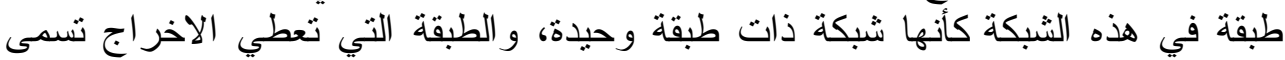

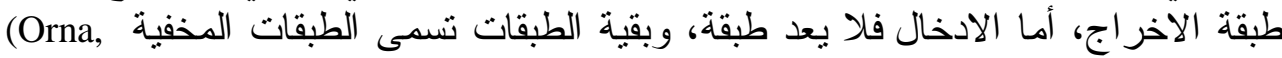
(2001 و الثكل الآتي يبين معمارية الثبكات ذات التئة التذذية الامامية:

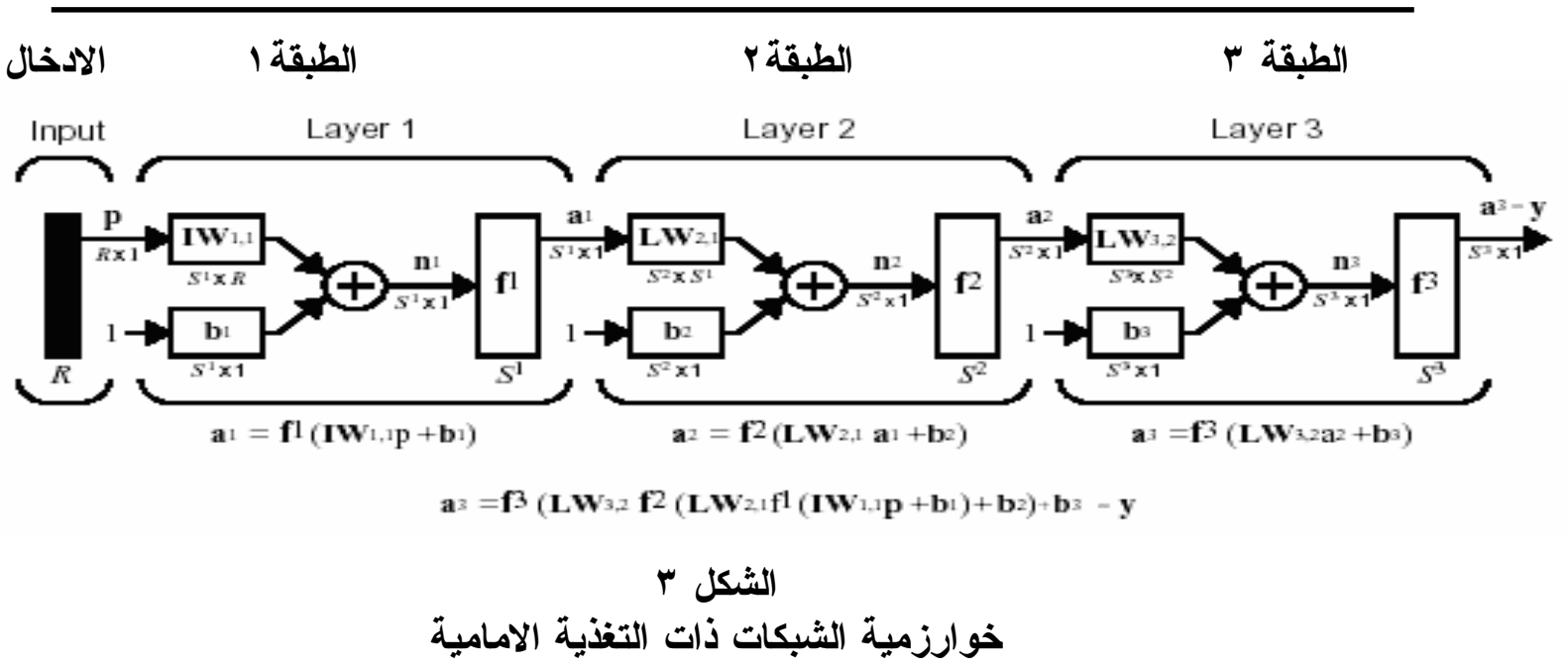

خوارزمية الثبكات ذات التفذية الامامية في تحليل الاحدار (Bacure, 1998)

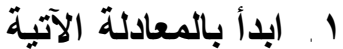
$\mathrm{p}=\delta\left(\sum_{\mathrm{i}=1}^{\mathrm{L}} \lambda_{\mathrm{i}} \delta\left(\mathrm{x} * \mathrm{w}_{\mathrm{i}}\right)\right)$

$$
\text { حيث إن: }
$$

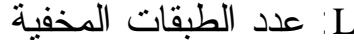

$$
\text { S(x)=1/1+ exp(-x) ويعبر عنها بالمعادلة Sigmoid }
$$


x : متجه الادخال (المتغير ات التوضيحية)

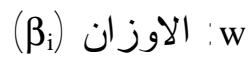

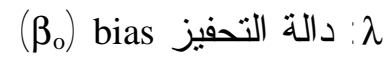

r ا ب بأخذ اللوغارتم تصبح المعادلة

$\log (\mathrm{p} /(1-\mathrm{p}))=\sum_{\mathrm{i}=1}^{\mathrm{L}} \lambda_{\mathrm{i}} \delta\left(\mathrm{x} * \mathrm{w}_{\mathrm{i}}\right)$

r. اثنقاق المعادلة بالنسبة الى x

$\frac{\partial}{\partial \mathrm{x}_{\mathrm{j}}} \log \left(\mathrm{p}(\mathrm{x}) /(1-\mathrm{p}(\mathrm{x}))=\sum_{\mathrm{i}=1}^{\mathrm{L}} \lambda_{\mathrm{i}} \delta\left(\mathrm{x}^{*} \mathrm{w}_{\mathrm{i}}\right) \mathrm{w}_{\mathrm{ij}}\right.$

ء. أما الاوزان فتحسب على وفق المعادلة

$\overline{\mathrm{w}}_{\mathrm{j}}(\mathrm{x})=\sum_{\mathrm{i}=1}^{\mathrm{L}} \lambda_{\mathrm{i}} \delta^{\prime}\left(\mathrm{x} * \mathrm{w}_{\mathrm{i}}\right) \mathrm{w}_{\mathrm{ij}}$

الجانب التطبيقي استخدمت بيانات عن مرضى سرطان الدم بعدد (سم) جمعت من المستشفى العام

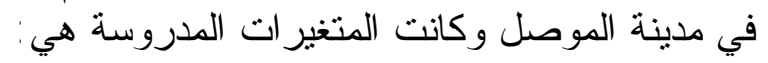
نسبة الخلايا السرطانية الجنس (ذكر ، انثى) X العمر X X الحالة الزوجية (اعزب، منزوج) X

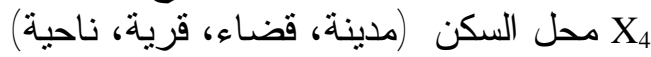
X الهيمو كلوبين X

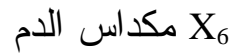
فحص دم له علاقة بالهيموكلوبين X

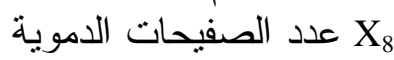
Xد $X_{9}$ X X

ا ـ تحليل الاحدار المتدرج Stepwise Regression

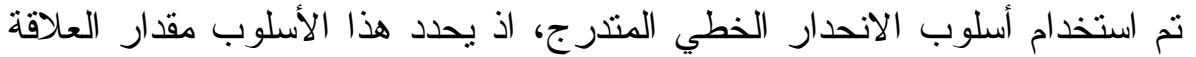

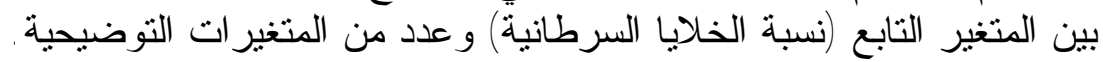

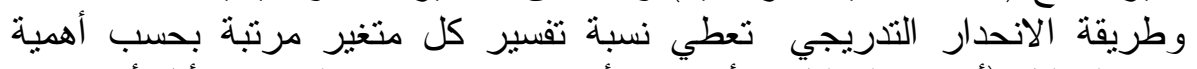
المتغير في التحليل (أي إن التحليل يبدأ بتحديد أهم متغير وينتهي بالمتغير الأقل أهمية في 
رشاد وزكي[ ·

$$
\text { في الجدول الاختلاف الذي يحدث في المتغير التابع). وتـــم الحصول على النتائج الموضحة }
$$

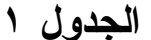

\section{جدول تحليل التباين باستخذام طريقة الاحدار التدريجي}

Stepwise Regression: y versus x1; x2; x3; x4; x5; x6; x7; x8; x9; x10

Alpha-to-Enter: 0.05 Alpha-to-Remove: 0.05

Response is y on 10 predictors, with $\mathrm{N}=53$

$\begin{array}{llll}\text { Step } & 1 & 2 & 3\end{array}$

Constant $27.42 \quad 46.17 \quad 53.57$

$\begin{array}{llll}\mathrm{x} 9 & 0.324 & 0.231 & 0.275\end{array}$

T-Value $\quad 4.20 \quad 2.85 \quad 3.56$

P-Value $\quad 0.000 \quad 0.006 \quad 0.001$

x7 $\quad-0.35-0.40$

T-Value $\quad-2.63-3.19$

P-Value $\quad 0.0110 .003$

$\mathrm{x} 10 \quad-20.7$

T-Value $\quad-2.90$

P-Value $\quad 0.006$

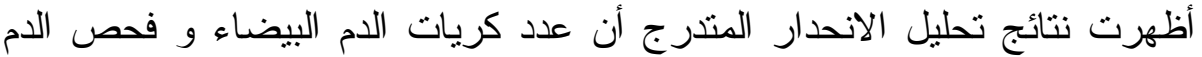

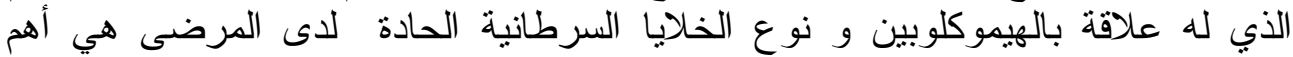

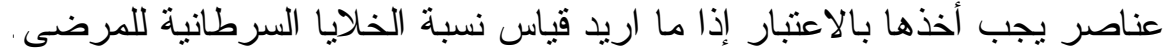

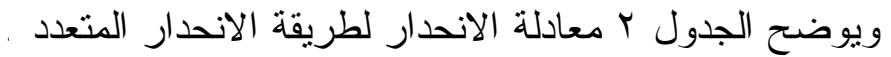

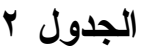 \\ معادلة الاحدار المتعدد}

The regression equation is

$y=53.6-0.400 \times 7+0.275 \times 9-20.7 \times 10$

$\begin{array}{lccccc}\text { Predictor } & \text { Coef } & \text { SE Coef } & \text { T } & \text { P } & \text { VIF } \\ \text { Constant } & 53.571 & 8.106 & 6.61 & 0.000 & \\ \text { x7 } & -0.4003 & 0.1256 & -3.19 & 0.003 & 1.3 \\ \text { x9 } & 0.27528 & 0.07728 & 3.56 & 0.001 & 1.3 \\ \text { x10 } & -20.656 & 7.133 & -2.90 & 0.006 & 1.1\end{array}$


استخدم برنامج حاسوبي لغرض التحليل باستخدام الثبكات العصبية الاصطناعية

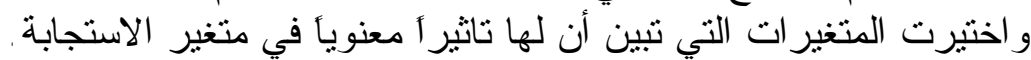

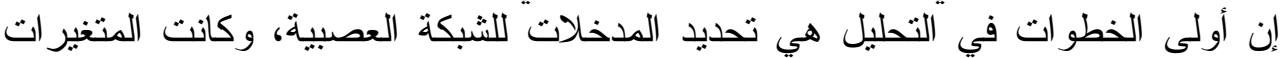

(عدد كريات الدم البيضاء، فحص الدام الذي لله علاقة بالهيموكلوبين ونوع الخلايا السرطانية) هي مدخلات الثبكة.

تم تحديد العقد في الطبقة المخفية و الذي يتحدد من خلال التدريب والعديد من التجارب الحاسوبية و الجدول ب يوضح مقدار الخطأ المطلق للشبكة.

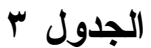

عدد العقد في الطبقة المخفية

\begin{tabular}{|c|c|c|}
\hline معمارية الشبكة & الخطظأ المطلق & الخطأ للشبكة \\
\hline $3-1-1$ & 17.199 & 0.059 \\
\hline $3-2-1$ & 15.384 & 0.044 \\
\hline $3-3-1$ & 17.242 & 0.051 \\
\hline $3-4-1$ & 17.529 & 0.052 \\
\hline
\end{tabular}

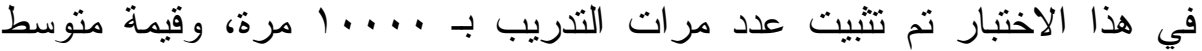

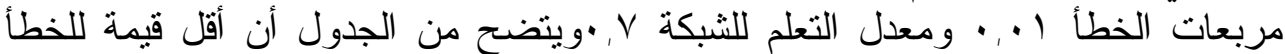
عند استخدام عصبونين للطبقة المخفية. تم تدريب شبكة عصبية ذات تغذية أمامية لحين الوصول الى أفضل شبكة عـصبية المبية

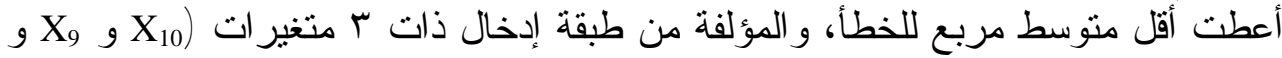

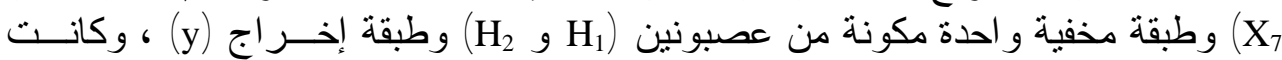

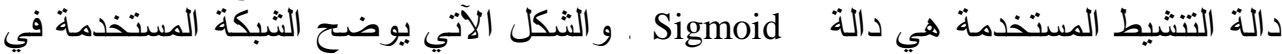
التحليل:

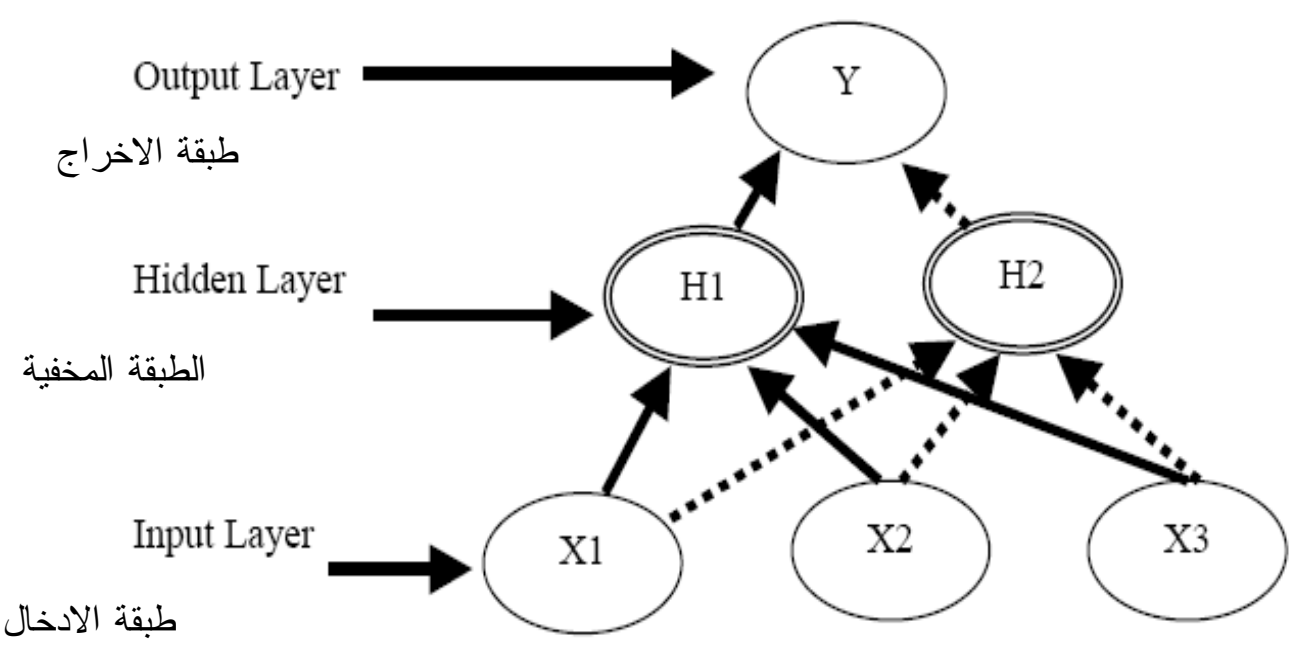


رشاد وزكي[r

\section{الثكل ؛}

الثبكة العصبية المستخدمة في تحليل الاحدار

ويوضح الجدول ع معادلة الانحدار وتحليل التبــاين لطريقــة الــشبكات العـصبية

الاصطناعية ذات التغذية الأمامية.

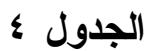 \\ معادلة الاحدار بطريقة الثبكات العصبية الاصطناعية}

The regression equation is

output $=60.9-0.426 \times 7+0.0909 \times 9-9.16 \times 10$

$\begin{array}{lccccc}\text { Predictor } & \text { Coef } & \text { SE Coef } & \text { T } & \text { P } & \text { VIF } \\ \text { Constant } & 60.918 & 5.694 & 10.70 & 0.000 & \\ \text { x7 } & -0.42610 & 0.08826 & -4.83 & 0.000 & 1.3 \\ \text { X9 } & 0.09088 & 0.05428 & 1.67 & 0.001 & 1.3 \\ \text { x10 } & -9.161 & 5.010 & -1.83 & 0.074 & 1.1\end{array}$

ويلاحظ من قيم VIF في الجدولين r و ع بان ليس هناك مشكلة تعـدد العلاقـات الخطية لكلتا الطريقتين.

ويوضح الجدول ه نتائج تحليل التباين لكل من طريقتي الانحدار المتعدد و الـشبكات العصبية الاصطناعية ذات التغذية الأمامية.

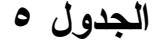 \\ ت تحليل التباين}

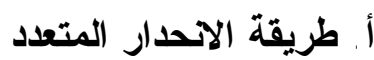

Analysis of Variance

\begin{tabular}{lrcclll} 
Source & DF & SS & MS & F & \multicolumn{2}{c}{ P } \\
Regression & 3 & 20737.2 & 6912.4 & 12.98 & 0.000 \\
Residual Error & 49 & 26087.6 & 532.4 & & \\
Total & 52 & 46824.8 & & & &
\end{tabular}

Analysis of Variance

ب. طريقة الثبكات العصبية الاصطناعية

$\begin{array}{llllll}\text { Source } & \text { DF } & \text { SS } & \text { MS } & \text { F } & \text { P }\end{array}$

$\begin{array}{llllll}\text { Regression } & 3 & 10144.4 & 3381.5 & 12.87 & 0.000\end{array}$

Residual Error $49 \quad 12872.1 \quad 262.7$

$\begin{array}{lll}\text { Total } & 52 & 23016.5\end{array}$ 
ومن مقارنة قيم MSe لكلتا الطريقتنين يتبــين بــأن طريقــة الـشيكات العــصبية

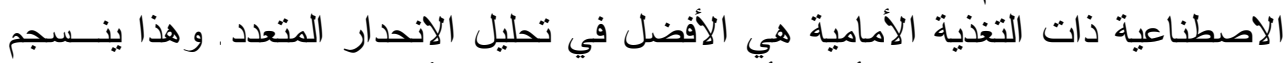

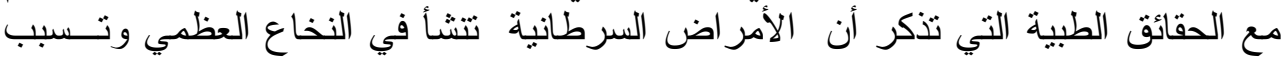

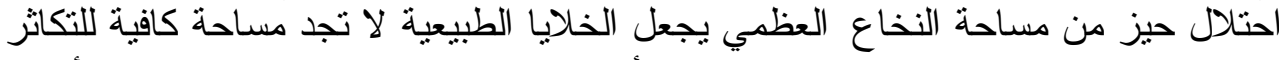

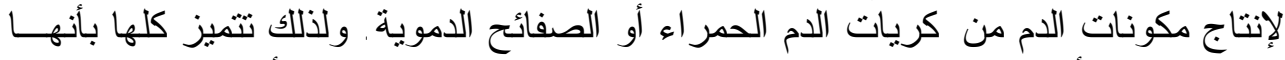

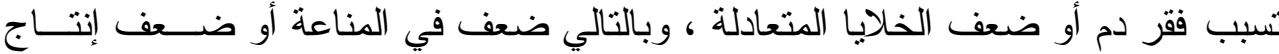

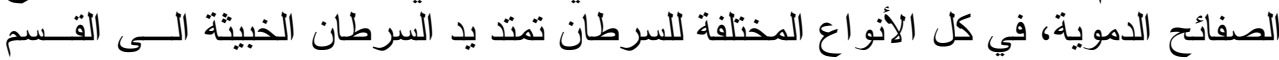

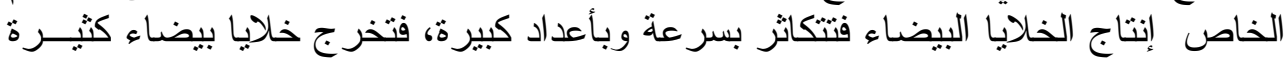

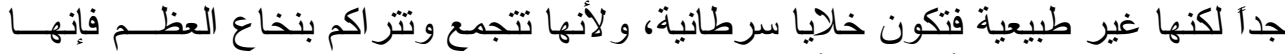

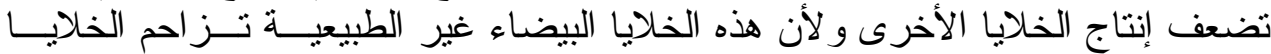

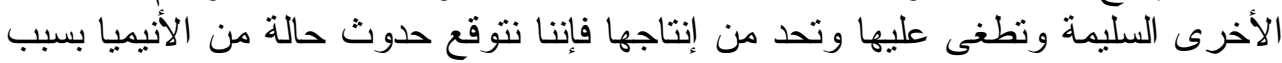

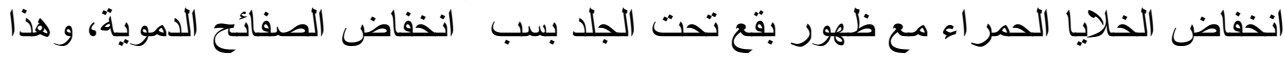

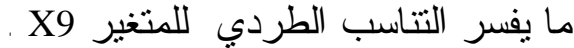
أما بالنسبة للمتغير X

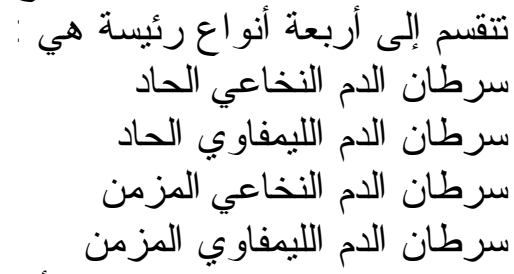

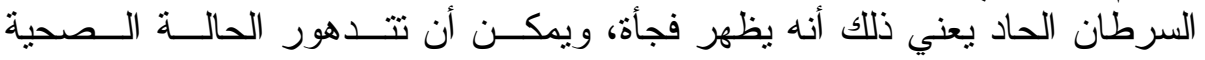

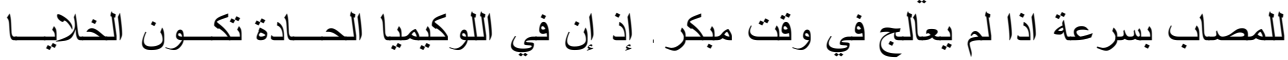

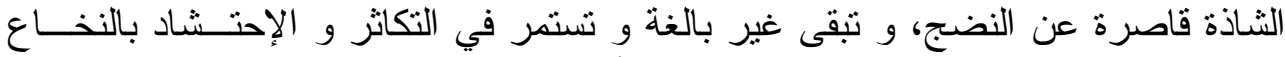

\begin{tabular}{|c|c|}
\hline \multicolumn{2}{|c|}{ 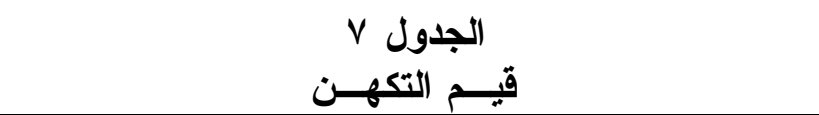 } \\
\hline التكهن بطريقة الثبكات العصبية & التكهن بطريقة الاحدار المتعدد \\
\hline 52.38 & 49.175 \\
\hline 56.214 & 68.5875 \\
\hline 60.048 & 91.3 \\
\hline 7.044 & 32.41 \\
\hline 32.57 & 16.275 \\
\hline 42.368 & 73.6 \\
\hline 40.664 & 51.1 \\
\hline 20.43 & 16.48 \\
\hline 47.054 & 32.4325 \\
\hline
\end{tabular}

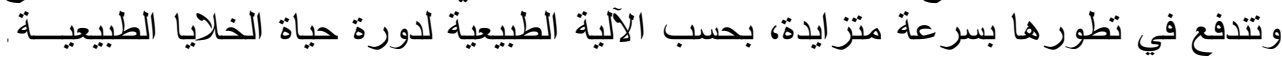

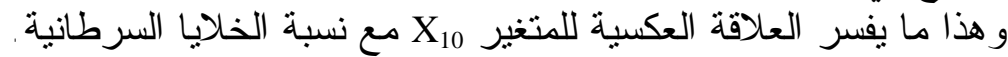

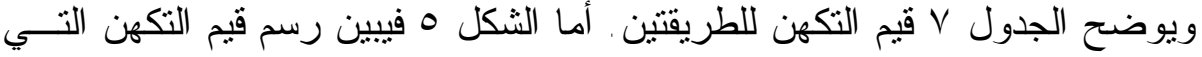

تم الحصول عليها للطريقتين. 
رشاد وزكي[؛ 1]

\begin{tabular}{|c|c|}
\hline التكهن بطريقة الشبكات العصبية & التكهن بطريقة الاتحدار المتعدد \\
\hline 26.82 & 32.985 \\
\hline 35.34 & 33.34 \\
\hline 26.18 & 13.6575 \\
\hline 52.38 & 53.85 \\
\hline 21.494 & 4.995 \\
\hline 20.642 & 24.875 \\
\hline 48.12 & 58.1 \\
\hline 35.34 & 30.315 \\
\hline 33.422 & 17.075 \\
\hline 57.066 & 73.1825 \\
\hline 16.808 & 1.2 \\
\hline 20.43 & 17.25 \\
\hline 53.658 & 49.3575 \\
\hline 38.534 & 23.525 \\
\hline 20.43 & 15.875 \\
\hline 28.95 & 24.975 \\
\hline 31.506 & 26.77 \\
\hline 34.488 & 29.625 \\
\hline 25.116 & 20.77 \\
\hline 51.528 & 51.675 \\
\hline 28.95 & 24.9475 \\
\hline 42.156 & 42.875 \\
\hline 26.82 & 23.8 \\
\hline 39.6 & 34.425 \\
\hline 46.842 & 55.14 \\
\hline 43.86 & 40.35 \\
\hline 32.358 & 28.175 \\
\hline 45.138 & 39.7625 \\
\hline 50.676 & 45.375 \\
\hline 40.664 & 29.1825 \\
\hline 47.906 & 34.8 \\
\hline 34.7 & 63.925 \\
\hline 26.82 & 25.725 \\
\hline 53.658 & 60.55 \\
\hline 26.82 & 22.2875 \\
\hline 35.552 & 18.8 \\
\hline 48.972 & 42.8675 \\
\hline 55.362 & 73.15 \\
\hline 56.64 & 54.825 \\
\hline 49.61 & 35.9875 \\
\hline 50.888 & 82.975 \\
\hline 39.6 & 34.975 \\
\hline 20.56 & 26.0975 \\
\hline
\end{tabular}




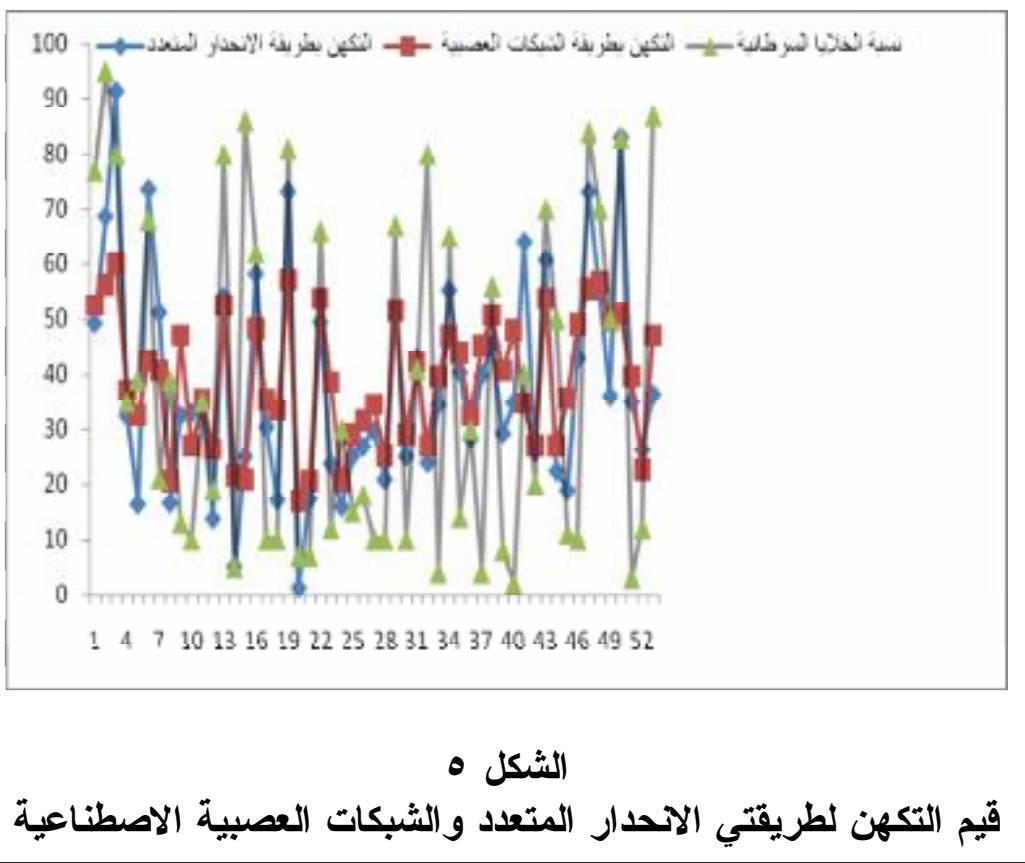

الاستنتاجات

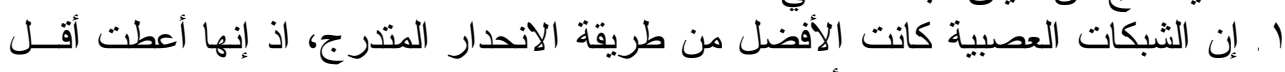

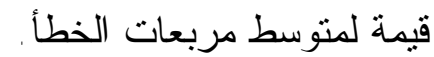
r. لاحظنا اختلاف نتائج الثبكات العصبية الاصنية التصناعية عن طريقة الانحدار التدريجي

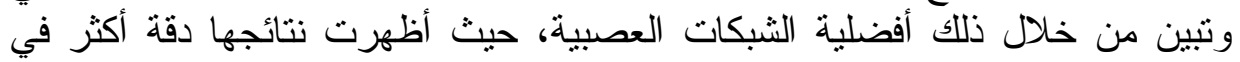
كثف المتغير ات ذات الفروقات غير المعنوية ـ وتتاسبت النتائج مع الحقائق الطبية . 
رشاد وزكي[14]

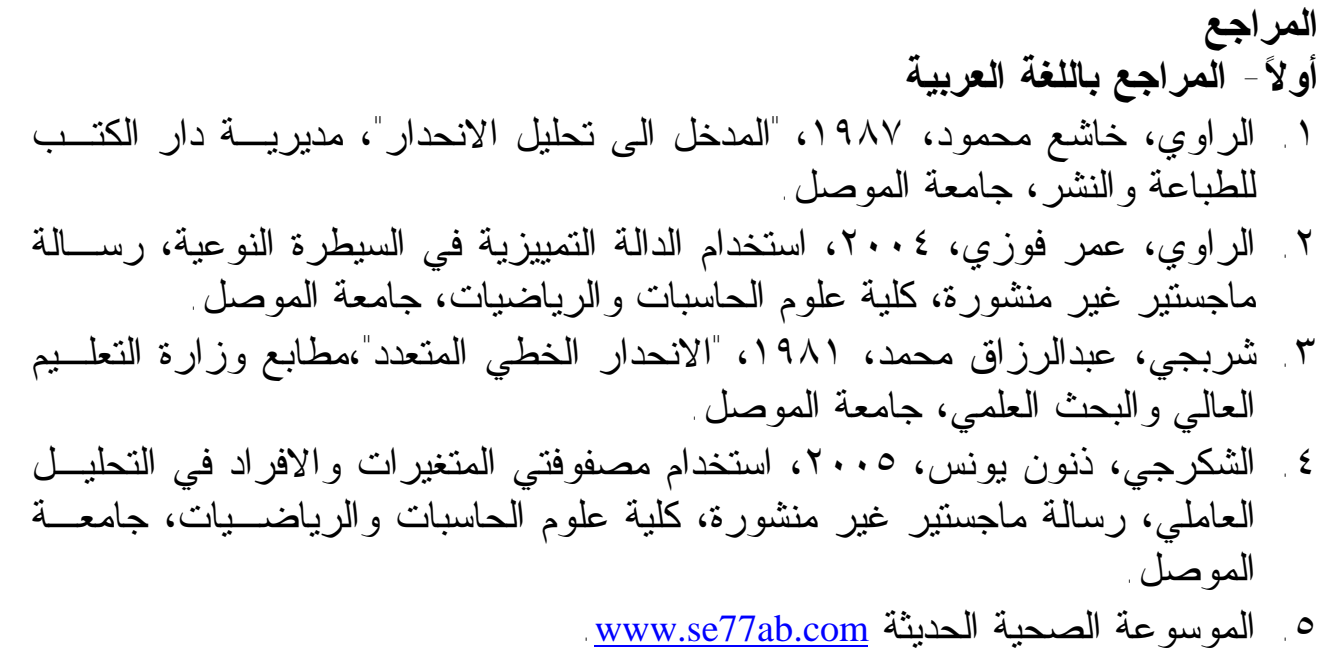

ثانياً - المر اجع باللغة الاجنبية

1. Bruce D. Baker, 1998, 'A Comparison of Linear Regression and Neural Network Methods for Forecasting Educational Spending', Department of Teaching and Leadership, 202 Bailey Hall, University of Kansas.

2. Orna Intrator and Nathan Intrator, 2001, "INTERPRETING NEURAL-NETWORK RESULTS: A SIMULATION STUDY", Brown University, Computer Science Department, February.

3. Wikipedia, The Free Encyclopedia, Stepwise regression, (2008),

http://en.wikipedia.org/wiki/Statistics. 